\title{
Clinical and laboratory awareness for an under recognized pathogen in newborn meningitis: Mycoplasma Hominis: a case report
}

\author{
Sinem Gülcan Kersin ${ }^{1 \oplus}$, Turgut Bozan ${ }^{2 \oplus}$, Hülya Özdemir ${ }^{1 \oplus}$, Hülya Selva Bilgen ${ }^{1 \oplus,}$ \\ Güner Söyletir ${ }^{2 \oplus}$, Aslı Memişoğlu $^{1 \oplus}$, Eren Özek ${ }^{1 \oplus}$ \\ ${ }^{1}$ Division of Neonatology, Departments of Pediatrics and ${ }^{2}$ Department of Medical Microbiology, Marmara University Faculty of \\ Medicine, İstanbul, Turkey.
}

\begin{abstract}
Background. Mycoplasma hominis is a well-known bacterium colonizing the genito-urinary tract. It may cause pneumonia, bacteremia, abscesses, chronic lung disease, and rarely meningitis during the newborn period.

Case. A preterm infant with a birth weight of 885 grams was born at 27 weeks of gestation and had respiratory distress syndrome needing mechanical ventilation. Spontaneous intestinal perforation and grade four intraventricular hemorrhage was diagnosed on day three.
\end{abstract}

Conclusion. M. hominis was accepted as the causative agent of meningitis in this case report.

Key words: Mycoplasma hominis, newborn, meningitis, neonate, preterm.

Mycoplasma hominis is a well-known bacterium colonizing the genito-urinary tract. $M$. hominis has been associated with a variety of conditions that may affect the pregnancy period, the developing fetusand thenewborn. Thisorganism may contribute to chorioamnionitis, growth restriction, spontaneous abortion, stillbirth, premature labor and postpartum endometritis. In addition, an exposed neonate may develop pneumonia, bacteremia, meningitis, abscesses and chronic lung disease. ${ }^{1}$ Mycoplasma hominis meningitis is a rare but life-threatening infection in both full term and preterm infants that might result in neurological deficits. ${ }^{2,3}$ Survivors frequently have long-term morbidity including severe neurodevelopmental impairment. In this case report, $M$. hominis meningitis in a preterm infant is discussed.

\section{Sinem Gülcan Kersin}

sinemgulcan@hotmail.com

Received 14th April 2019, revised 16th May 2019, accepted 27th May 2019.

\section{Case Report}

A 27-year-old G2P1 female at 27 weeks of gestation was admitted to the hospital with fever and coughing. On admission she developed acute respiratory distress and was transferred to the intensive care unit with the preliminary diagnosis of acute respiratory failure and sepsis. The patient was placed on mechanic ventilation, cardiac inotropic agents, large spectrum antibiotics and steroids were administered. No evidence of infection in blood and endotracheal aspirate (ETA) cultures was obtained except Candida albicans in the urine culture. As the patient clinically deteriorated despite the supportive measures and she had premature rupture of membranes, the infant was delivered with induction of labor in the intensive care unit. A female infant with a birth weight of 885 grams was born at 27 weeks of gestation with Apgar scores of 2, 3 and 5 at 1, 5 and 10 minutes respectively. Surfactant was administered and the infant was admitted to the neonatal intensive care unit (NICU). Blood and ETA cultures were obtained and vancomycin and meropenem 
therapy were started due to the high-risk birth history. She was on fluconazole prophylaxis, caffeine and total parenteral nutrition and because of hypercapnia and low blood partial oxygen saturation; respiratory support was changed to high frequency oscillatory ventilation. On day two of life, liposomal amphotericin B was added to the treatment due to progressively increasing acute phase reactants, newly developed thrombocytopenia and inadequate clinical improvement under current therapy. The infant subsequently developed sinus tachycardia and morphine infusion was initiated for pain management. However, the infant's tachycardia persisted. On postnatal day three, greenish discoloration of the abdomen and general pallor evolved within minutes and spontaneous intestinal perforation was observed on abdominal X-Ray. The infant became hypotensive, with prolonged capillary filling time and bolus saline infusions were administered. Hemoglobin value was found to be $6.9 \mathrm{~g} / \mathrm{dl}$ and and erythrocyte transfusion was given. Grade IV intraventricular hemorrhage was detected on transfontanel ultrasonography. The infant developed seizures on the third day and phenobarbital was administered, and levetiracetam was added to the therapy for persistent seizures. Bilateral extraventricular drains were placed by pediatric neurosurgery on day seven of life. On day 10 of triple antimicrobial therapy, there was still significant increase in acute phase reactants. Blood, ETA, cerebrospinal fluid (CSF) and urine cultures were obtained and colistin was added to therapy. No growth was detected in the blood, ETA, urine and CSF cultures. Colistin and liposomal amphotericin B were discontinued. However; acute phase reactants including CRP levels and leucocyte counts, continued to increase from $8 \mathrm{mg} / \mathrm{L}$ to $113 \mathrm{mg} / \mathrm{L}$ and $7600 / \mu \mathrm{L}$ to $33900 / \mu \mathrm{L}$, respectively. On the 17th of life, second CSF culture was submitted to the microbiology laboratory. Leucocytes and erythrocytes with no microorganism were seen on Gram stained preparation of the sample. CSF was also inoculated onto blood agar, chocolate agar and thioglycollate broth and inoculated media were followed up daily for five days. Translucent and pinpoint colonies weakly grew on chocolate agar and blood agar on fifth day of incubation. No organism was observed on the Gram stained slide prepared from those colonies. Upon this, subculture from thioglycollate broth was performed. Two days later, translucent colonies were seen again on chocolate agar and this microorganism was identified as Mycoplasma hominis with MALDI-TOF MS. Since M. hominis is known generally as a cell wall deficient bacterium and require special medium to grow, to confirm our result, $16 \mathrm{~S}$ rRNA gene sequencing was also performed directly from CSF sample of the patient. Genomic DNA was extracted from CSF by using DNeasy Blood and Tissue kit (Qiagen). 16S rRNA gene was amplified by PCR using universal primers $8 \mathrm{UA}$ and $907 \mathrm{~B}$ and subsequently sequenced with an ABI Prism 3100 Genetic Analyser (Applied Biosystems, Inc.). The BLAST software was used to search for DNA nucleotide sequences against similar nucleotide sequences in the database. ${ }^{4}$ The $16 \mathrm{~S}$ rRNA gene sequence showed $99 \%$ nucleotide identity to that of the strain $M$. hominis (GenBank accession no: CP033021.1). M. hominis was accepted as the causative agent of meningitis and antibiotic therapy was changed to ciprofloxacin monotherapy. On the second day of ciprofloxacin treatment, acute phase reactants decreased and therapy was continued for 21 days. On day 50 of life, the MRI showed multicyctic porencephalic hydrocephaly. The Auditory Brainstem Response (ABR) test was within normal limits. Informed consent was received from the family for writing this case. The patient was discharged at the end of the third month with a transpyloric feeding catheter and ventriculoperitoenal (VP) shunt. He had moderate neurological impairment including headlag, truncal hypotonia, unresponsiveness to sounds and there was also social developmental delay according to the Denver II test.

\section{Discussion}

Mycoplasma hominis is a rare but life-threatening infection in both full term and preterm 
infants. M. hominis may be the causative agent of bacteremia, pneumonia, meningitis in newborns, but its significance as a pathogen causing neonatal meningitis is still unclear. M. hominis can also be isolated in CSF from infants without signs of meningitis. ${ }^{5}$ Therefore, the clinical findings in newborns with $M$. hominis isolated from the CSF are variable. It may produce abnormal CSF findings such as pleocytosis or sometimes there is no inflamatory reaction in CSF.

Mycoplasma species are small-sized microorganisms. There are more than 100 species of Mycoplasma and at least 13 Mycoplasma species have been known to infect humans. Mycoplasma pneumoniae, M. hominis and Ureaplasma urealyticum are the most wellknown species. ${ }^{6}$ The lack of bacterial cell wall components makes $M$. hominis undetectable by Gram staining. Growth on bacterial cultures is very slow, requires specific media and occasionally can be found as pinpoint colonies on conventional media. ${ }^{7}$ In our case; cerebrospinal fluid culture obtained on postnatal day 17 revealed pinpoint, translucent colonies on fifth day of incubation on all inoculated media and subcultures and Mycoplasma hominis was identified with MALDI-TOF MS. Furthermore, $16 \mathrm{~S}$ rRNA gene sequencing of the sample also revealed presence of $M$. hominis.

M. hominis is intrinsically resistant to many antibiotics such as B-lactams, glycopeptides, sulfonamides and macrolides. The major antibiotics active against $M$. hominis are the tetracyclines, lincosamides, chloramphenicol and fluoroquinolones. ${ }^{8}$ Our case did not respond to the broad spectrum antimicrobials but responded to ciprofloxacin within two days with a significant decline in CRP levels and total leucocyte count.

M. hominis is found in the urogenital tract with a prevalence between $15-17.7 \%$ and is mainly involved in urogenital infections and neonatal infections. ${ }^{910}$ In our case the mother did not have a good follow-up during pregnancy and neither vaginal cultures nor screening for GBS was performed. Approximately half of the women with this infection may be asymptomatic or may experience only mild symptoms. In our case, the mother developed acute respiratory failure and sepsis at the $27^{\text {th }}$ week of gestation. All her cultures including blood, urine and ETA cultures during her ICU stay, remained sterile. Placental pathology revealed chorioamnionitis but the cultures remained sterile as she was already receiving broad spectrum antibiotics. The mechanism by which bacterial vaginosis may lead to preterm birth is unknown. ${ }^{11}$

Simhan et al. ${ }^{12}$ explains that ascending intrauterine infection occurs because of alterations in host-defense mechanisms and microbial factors. Since individuals differ in their innate ability to create an inflammatory response to bacterial products, it has been suggested that women who are immunologically hyporesponsive may not be able to control a large bacterial burden and are prone to ascending infection with variable consequences. The history of the mother revealed that she had isolated watery vaginal discharge with fishy odor about one month prior to presentation which might be the reason for her preterm birth.

In the literature extragenital infections such as joint, bone and CNS infections by M. hominis have been described, especially in newborn infants and immunocompromised patients. However, there are few reported cases of $M$. hominis meningitis in either premature or full term infants. ${ }^{13}$ Until now this is also our first case diagnosed with $M$. hominis meningitis in our NICU. Hata et al. ${ }^{2}$ reported that about one third of their adult patients with M. hominis meningitis had CNS complications and $28 \%$ of them had permanent neurological sequelae and $28 \%$ of them died. In this case, our patient underwent VP-shunt operation at $90^{\text {th }}$ day of life after being diagnosed with hydrocephalus due to intraventricular bleeding on the postnatal day 10. During this period, ventricular taps were performed twice a week to decrease intracranial pressure. 
Vertical transmission to both premature and full term infants may occur during labor. In a study by Chua et al. ${ }^{10,11}$ the transmission rate to infants was $30 \%$ for $M$. hominis. As the mother did not have any vaginal cultures or screening before birth and soon afterwards, we were not able to prove that it was transmitted from the mother.

In conclusion, as shown in our case report, in clinical practice, when neonates have clinical and laboratory signs of sepsis/meningitis and deteriorate despite broad spectrum antimicrobials, microbiological evaluation is of prime importance. Incubation period of culture media must be at least five days for CSF sample and colonies must be evaluated carefully for microorganisms which are difficult to grow in routine culture media.

\section{REFERENCES}

1. Waites KB, Schelonka RL, Xiao L, Grigsby PL, Novy MJ. Congenital and opportunistic infections: ureaplasma species and Mycoplasma hominis. Semin Fetal Neonatal Med 2009; 14: 190-199.

2. Hata A, Honda Y, Asada K, Sasaki Y, Kenri T, Hata D. Mycoplasma hominis meningitis in a neonate: case report and review. J Infect 2008; 57: 338-343.

3. Watt KM, Massaro MM, Smith B, Cohen-Wolkowiez M, Benjamin Jr DK, Laughon MM. Pharmacokinetics of moxifloxacin in an infant with Mycoplasma hominis meningitis. Pediatr Infect Dis J 2012; 31: 197-199.

4. Benson DA, Boguski MS, Lipman DJ, Ostell J. GenBank. Nucleic Acids Res 1997; 25: 1-6.
5. Wolthers KC, Kornelisse RF, Platenkamp GJJM, et al. A case of Mycoplasma hominis meningoencephalitis in a full-term infant: rapid recovery after start of treatment with ciprofloxacin. Eur J Pediatr 2003; 162: 514-516.

6. Sethi S, Singh G, Samanta P, Sharma M. Mycoplasma genitalium: an emerging sexually transmited pathogen. Indian J Med Res 2012; 136: 942-955.

7. Siber GR, Alpert S, Smith AL, Lin JSL, McCormack WM. Neonatal central nervous system infection due to Mycoplasma hominis. J Pediatr 1977; 90: 625-627.

8. Reissier S, Masson R, Guérin F, et al. Fatal nosocomial meningitis caused by Mycoplasma hominis in an adult patient: case report and review of the literature. Int J Infect Dis 2016; 48: 81-83.

9. Sobouti B, Fallah S, Mobayen M, Noorbakhsh S, Ghavami Y. Colonization of Mycoplasma Hominis and Ureaplasma u r e a l y t i c u m in pregnantwomen and their transmission to offspring. Iran J Microbiol 2014; 6: 219-224.

10. Chua KB, Ngeow YF, Lim CT, Ng KB, Chye JK Colonization and transmission of Ureaplasma urealyticum and mycoplasma hominis from mothers to full and preterm babies by normal vaginal delivery. Med J Malaysia 1999; 54: 242-246.

11. Chua KB, Ngeow YF, Ng KB, Chye JK, Lim CT. Ureaplasma urealyticum and Mycoplasma hominis isolation from cervical secretions of pregnant women and nasopharyngeal secretions of their babies at delivery. Singapore Med J 1998; 39: 300-302.

12. Simhan HN, Caritis SN, Krohn MA, de Tejada BM, Landers DV, Hillier SL. Decreased cervical proinflammatory cytokines permit subsequent upper genital tract infection during pregnancy. Am J Obstet Gynecol 2003; 189: 560-567.

13. Watson L, Min Pang Y, Mitchell S, Dodgson A. Mycoplasma hominis meningitis in a 24 week premature neonate: case report and short literature review. J Pediatr Pharmacol Ther 2008; 13: 251-254. 